\begin{tabular}{|c|c|c|}
\hline & International Journal of Basic and Applied Sciences, $7(1)(2018) 1-7$ & \\
\hline & International Journal of Basic and Applied Sciences & \\
\hline & $\begin{array}{c}\text { Website: www.sciencepubco.com/index.php/IJBAS } \\
\text { doi: } 10.14419 / \text { ijbas.v7il.8633 }\end{array}$ & 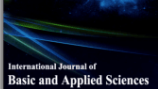 \\
\hline & Research paper & \\
\hline
\end{tabular}

\title{
Hysteresis, Quasiperiodicity and Chaoticity in a Nonlinear Dissipative Hybrid Oscillator
}

\author{
A. V. Monwanou ${ }^{1 *}$, C. H. Miwadinou ${ }^{2}$, C. Ainamon ${ }^{3}$ and J. B. Chabi Orou ${ }^{3}$ \\ ${ }^{1}$ Institut de Mathématiques et de Sciences Physiques, Université d'Abomey-Calavi, Bénin \\ ${ }^{2}$ Ecole Normale Supérieure de Natitingou, Université d'Abomey, Bénin \\ ${ }^{3}$ Institut de Mathématiques et de Sciences Physiques, Université d'Abomey-Calavi, Bénin \\ ${ }^{4}$ Institut de Mathématiques et de Sciences Physiques, Université d'Abomey-Calavi, Bénin \\ *Corresponding author E-mail: movins2008@yahoo.fr
}

\begin{abstract}
Hysteresis, quasiperiodicity and chaoticity in a nonlinear dissipative hybrid oscillator are studied. The modified Rayleigh-Duffing oscillator is considered. We simultaneously take into account the new nonlinear cubic, pure quadratic and hybrid dissipative terms which modify the classical Rayleigh-Duffing oscillator. The influence of each of these new parameters on the dynamics of the oscillator has been seriously studied and interesting results are obtained. It is clear that each of these new dissipation terms can be used to control the dynamics of this oscillator. Some may be used to reduce or eliminate hysteresis, amplitude jump and resonance phenomena; others may accentuate them. Similarly, these new parameters can be used to impose on the systems modeled by this oscillator, a regular, quasi-periodic or even chaotic behavior according to their field of application. Thus, one of the original results obtained is the equation of the curve delimiting the zone of instabilities of the amplitudes of harmonic oscillations. This equation thus makes it possible to know the zone of amplitude permitted or of the amplitude jump for the systems and thus to control and predict the loss or gain of energy during this jump. Finally, the second stability of the oscillations of the system is studied as well as the influence of the dissipation parameters on this stability. It should be noted that the influence of some of these parameters depends on the simultaneous presence of these parameters.
\end{abstract}

Keywords: chaos; Floquet theory; hysteresis; Modified Rayleigh-Duffing oscillator; Whittaker method.

\section{Introduction}

In recent years, a twofold interest has attracted theoretical, numerical, and experimental investigations to understand the behaviour of nonlinear oscillators. Theoretical (fundamental) investigations reveal their rich and complex behaviour, and the experimental (self-excited oscillators) describes the evolution of many biological, chemical, physical, mechanical, and industrial systems $[1,2]$. The interest devoted to chaos by many scientist is due to the fact that this new phenomenon appears in various fields, from mathematics, physics, biology, and chemestry, to engineering, economics and medecine. Consequently, there are many opportunities for application of chaos. For example, in physics, chaos has been used to refine the understanding of planetory orbits, to reconceptualize quantum level processes, and to forecast the intensity of solar activity. In engineering, chaos has been used in bulding of better digital filters, and to model the structural dynamics in such structures as buckling columns. In medecine, it has been used to study cardiac arrhythmias and patterns of disease communication. In psychology, it has been used to study mood fluctuations, the operation of the olfactory lobe during perception, and partterns of innovation in organizations. In economic it is being used to find patterns and develop new types of econometric model for the stack market to variations in coton prices. There are also many opportunities for exploitation of chaos: synchronized chaos, mixing with chaos, encoding information with chaos, anti-control of chaos, tracking of chaos and targetting of chaos.

An important class of systems in general and in particular oscillators who presented a complex or chaotic behaviour can be determined on the basis of nonlinear damping [3]. Such damping can, in some systems, change the sign depending on velocity or displacement values, and provide excitation energy to the examined system. These, so called, self-excited damping terms are often used to describe systems with dry friction, bearings lubricated by a thin layer of oil, shimming in vehicle wheels or chatter in a cutting process $[4,5,6,7]$. In Ref. [8], the authors have studied with considerable detail the effects of the damping level on the resonance response of the steadystate solutions and in the basin bifurcation patterns of the escape oscillator. In particular they analyzed the effect of using different damping levels and how this contributes to the erosion of the safe areas in phase space, and they also provided a comprehensive global picture of the main bifurcation boundaries. More recently such a nonlinear damping force has also been considered [9] of a modern vehicle suspension system due to electro- or magneto-rheological fluid damping where it is causing a hysteretic effect. In this model, the authors used a self-excited term of the Rayleigh and the Duffing type with a double well potential. Parametric excitation occurs in a wide variety of engineering application [10].

In this vein, we propose to study in this paper hysteresis, quasiperiodicity and chaoticity in a system modeled by a modified Rayleigh- 
Duffing oscillator on the form

$$
\begin{array}{r}
\ddot{x}+\mu\left(1-\dot{x}^{2}\right) \dot{x}+\beta \dot{x}^{2}+k_{1} \dot{x} x+k_{2} \dot{x}^{2} x+ \\
(\gamma+\alpha \cos \Omega t) x+\lambda x^{3}=F \cos \Omega t,
\end{array}
$$

where $\mu, \beta, k_{1}, k_{2}, \gamma, \lambda, F$ and $\Omega$ are parameters. Physically, $\mu, k_{2}, \beta$ and $k_{1}$ represent respectively pure cubic, unpure cubic, pure quadratic and unpure quadratic nonlinear damping coefficient terms; $\alpha$ and $F$ are respectively the amplitudes of the parametric and external periodic forcing whereas $\sqrt{\gamma}$ and $\Omega$ are respectively natural and external forcing frequency. Moreover $\lambda$ characterizes the intensity of the restoring nonlinearity. The nonlinear damping term corresponds to the Modified Rayleigh oscillator, while the nonlinear restoring force corresponds to the Duffing oscillator. The basic physical sense of the Rayleigh-Duffing equation lies in the allowance for the dependence on the highest powers of the velocity in the dissipative coefficient and in the frequency for a classical oscillator.

This equation which has nonlinear dissipative terms and parametric excitation term can be used to model some systems such as Brusselator, Selkov, rolling response, certain MEMS systems, El Ninosouthern oscillation... [4, 11, 12, 13, 14, 15, 16, 17, 18, 19, 20].

The paper is organized as follows. In Section 2, we focussed a deph attention on harmonic oscillatory states and stability analysis in order to study hysteresis and jump phenomena. Section 3 addresses the phase portraits, largest Lyapunov exponent, the bifurcation diagrams and basins of attraction, from which a concluding remark is made in connection to the tendency of the system to have quasiperiodic, nonperiodic evolutions and the transition to chaos according to the choice of initial conditions. We provided a conclusion in the last section.

\section{Harmonic oscillatory states and stability analysis}

\subsection{Harmonic oscillatory states}

Assuming that the fundamental component of the solution and the external excitation have the same period, the amplitude of harmonic oscillations can be tackled using the harmonic balance method [5, $21,22,23]$. For this purpose, we express its solutions as

$$
x=A \cos (\Omega-\psi) t+\xi
$$

where $A$ represents the amplitude of the oscillations and $\xi$ a constant Inserting this solution Eq.(2) into Eq.(1) and equating the constants and the coefficients of $\sin \Omega t$ and $\cos \Omega t$, we have

$$
\begin{aligned}
& {\left[-A \Omega^{2}+\frac{1}{2} \beta \Omega^{2} A^{2}+\alpha \xi+\gamma A+3 \lambda \xi^{2} A+\right.} \\
& \left.\left[\frac{3}{4} \lambda A^{3}-\frac{1}{4} k_{2} \Omega^{2} A^{3}\right]^{2}++\mu \Omega A-\frac{3}{4} \mu \Omega^{3} A^{3}+k_{1} \Omega \xi A\right]^{2} \\
& =F_{0}^{2},
\end{aligned}
$$

$-\frac{1}{2} \beta \Omega^{2} A^{2}-\frac{1}{2} k_{2} \xi \Omega^{2} A^{2}+\frac{1}{2} \alpha A+\gamma \xi+\lambda \xi^{3}+\frac{3}{2} \lambda \xi A^{2}=0$.

If it is assumed that $|\xi| \ll|A|$, then $\xi^{2}$ and $\xi^{3}$ terms can be neglected and Eq.(4) becomes

$-\frac{1}{2} \beta \Omega^{2} A^{2}-\frac{1}{2} k_{2} \xi \Omega^{2} A^{2}+\frac{1}{2} \alpha A+\gamma \xi+\frac{3}{2} \lambda \xi A^{2}=0$

which solution is

$\xi=\frac{-\frac{1}{2} \beta \Omega^{2} A^{2}+\frac{1}{2} \alpha A}{-\gamma-\frac{1}{2}\left(-k_{2} \Omega^{2}+3 \lambda\right) A^{2}}$.
Substituting the solution Eq.(6) into Eq. (3) one obtains the following nonlinear algebraic equation

$$
\begin{gathered}
{\left[-A \Omega^{2}+\frac{1}{2} \beta \Omega^{2} A^{2}+\gamma A+A+\frac{1}{4}\left(-k_{2} \Omega^{2}+3 \lambda\right) A^{3}+\right.} \\
\left.\frac{-\frac{1}{2} \alpha \beta \Omega^{2} A^{2}+\frac{1}{2} \alpha^{2} A}{-\gamma-\frac{1}{2}\left(-k_{2} \Omega^{2}+3 \lambda\right) A^{2}}\right]^{2}+ \\
{\left[\mu \Omega A-\frac{3}{4} \mu \Omega^{3} A^{3}+k_{1} \Omega \frac{-\frac{1}{2} \beta \Omega^{2} A^{2}+\frac{1}{2} \alpha A}{-\gamma-\frac{1}{2}\left(-k_{2} \Omega^{2}+3 \lambda\right) A^{2}} A\right]^{2}} \\
=F_{0}^{2} .
\end{gathered}
$$

We investigate the effects of the different parameters on the oscillation amplitude $A$. For this purpose, we solved Eq. (7) using the Newton-Raphson algorithm. Fig.1 shows the amplitude-response curve where hysteresis and jump phenomena appear. Figs.1 $(a)$ and (b) illustrate the effect of the pure cubic damping and pure quadratic damping on amplitude-response curve respectively. Through these figures, we notice that jump and hysteresis phenomena appear when each damping coefficient increases. In Fig.1 $(c)$, the effect of the amplitude of parametric excitation $\alpha$ is shown. These two phenomena always exist whatever the value of $\alpha$ and become more and more accentuated when $\alpha$ increases. The behaviour of the amplitude of the system oscillations is investigated when the external frequency $\Omega$ varies. The analytical and numerical frequency-response curves obtained are provided in Fig.2. The resonance obtained from Fig.2 is affected by the nonlinear damping parameter, the nonlinear restoring parameter, the parametric excitation amplitude and the external forced amplitude (see Figs. 3, 4). Hysteresis and jump phenomena, and multistability oscillations are also observed and are affected by the nonlinearities parameters and parametrical excitation amplitude. For instance, from Figs.3 $(a)$ and $(b)$ the peak value of resonance amplitude increases while the resonance frequency descreases with the external excitation amplitude $F$ and parametrical excitation amplitude $\alpha$. The resonance amplitude increases while the resonance frequency decreases when the nonlinear restoring parameter $\lambda$ increases remaining below $\lambda=1$ (see Fig. 3(c)); the inverse evolutions are observed for the amplitude and the resonant frequency when $\lambda$ increases from $\lambda=1$ (see Fig. $3(d)$ ). In Figs.4(a), $(b)$ and $(d)$, we noticed that the unpure cubic damping $k_{2}$ has the same effect as $\lambda$ while the pure cubic damping $\mu$ and unpure quadratic damping $k_{1}$ have the opposite one. From Fig.4(c), it can been pointed out that when $\beta$ increases, the resonance amplitude decreases while the resonance frequency increases and hysteresis appears.

Fig. 5 shows the jump and hysteresis phenomena for a given value of restoring amplitude $\lambda=3.5$ at different levels of external excitation. Here, we obtained the loci of the two sets of jump points for different values of $F$. The equations of these two loci can be obtained as discussed below. At the points of vertical tangency in Fig. 5, $\frac{d A}{d \Omega} \rightarrow$ $\infty$. Differentiating both sides of Eq. (7) with respect to $\Omega$ with other parameters as constants and setting the denominator for $\frac{d A}{d \Omega}$ to zero, gives

$$
\begin{aligned}
& \delta_{1} \Delta_{1} D^{2}+\delta_{2} \Delta_{2} D^{2}+\left(-\beta \Omega^{2} A+\frac{1}{2} \alpha\right) D+ \\
& \left(3 \lambda-k_{2} \Omega^{2}\right) A N=0
\end{aligned}
$$

with

$$
\begin{gathered}
N=-\frac{1}{2} \beta \Omega^{2} A^{2}+\frac{1}{2} \alpha A, \\
D=-\gamma-\frac{1}{2}\left(3 \lambda-k_{2} \Omega^{2}\right) A^{2}, \\
\delta_{1}=-\Omega^{2}+\beta \Omega^{2} A+\gamma+\frac{9}{4} \lambda A^{2}-\frac{3}{4} k_{2} \Omega^{2} A^{2}, \\
\delta_{2}=\mu \Omega-\frac{9}{4} \mu \Omega^{3} A^{2}+k_{1} \Omega \xi,
\end{gathered}
$$



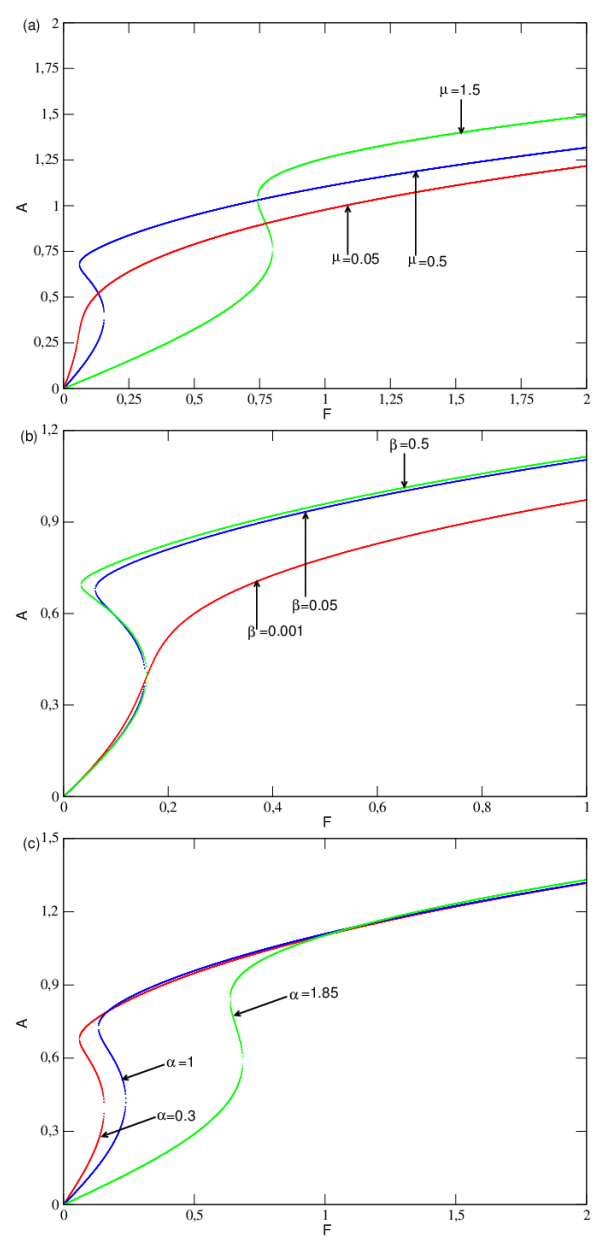

Figure 1: Amplitude-response curves with the parameters $\alpha=0.3, \beta=$ $0.05, k_{1}=0.5, k_{2}=0.5, \mu=0.5, \lambda=1, \gamma$ and $\Omega=1.105$. (a) Effect of $\mu$, (b) Effect of $\beta,(c)$ Effect of $\alpha$

$$
\begin{gathered}
\Delta_{1}=-\Omega^{2} A+\frac{1}{2} \beta \Omega^{2} A^{2}+\alpha \xi+\gamma A+\frac{3}{4} \lambda A^{3}-\frac{1}{2} k_{2} \Omega^{2} A^{3}, \\
\Delta_{2}=\mu \Omega A-\frac{3}{4} \Omega^{3} A^{3}+k_{1} \Omega \xi A .
\end{gathered}
$$

Thus, Eq. (8) describes the two loci of the jump points.

\subsection{Stability analysis}

Stability analysis of the harmonic solution given by Eq. (7) is now carried out. A convenient analytical tool for addressing this issue of stability is the Floquet theory [5, 22, 23]. For this, the harmonic solution is perturbed by $\xi$ and the time development of this disturbance is tracked. Thus, $x=A \cos (\Omega-\psi) t+\xi$ is substituted into Eq. (1). By setting $\tau=\frac{\Omega t-\psi}{2}$ and neglecting nonlinear terms of $\xi$ and the constant terms, we obtain the following linear variational equation :

$$
\ddot{\xi}+[2 \Upsilon+\Phi(\tau)] \dot{\xi}+\Xi(\tau) \xi=0,
$$

where

$$
\Upsilon=-\varepsilon+\frac{\mu}{2}-\mu \frac{4 k_{2} A^{2}}{\Omega}
$$

$\Phi(\tau)=\Gamma_{1} \cos 2 \tau+\Gamma_{2} \sin 2 \tau+\Gamma_{3} \cos 4 \tau+\Gamma_{3} \sin 4 \tau$,

$\Xi(\tau)=\Lambda_{0}+\Lambda_{1} \cos 2 \tau+\Lambda_{2} \sin 2 \tau+\Lambda_{3} \cos 4 \tau+\Lambda_{4} \sin 4 \tau$, with

$$
\Gamma_{1}=k_{1} A, \quad \Gamma_{2}=-\frac{8 \beta A}{\Omega},
$$

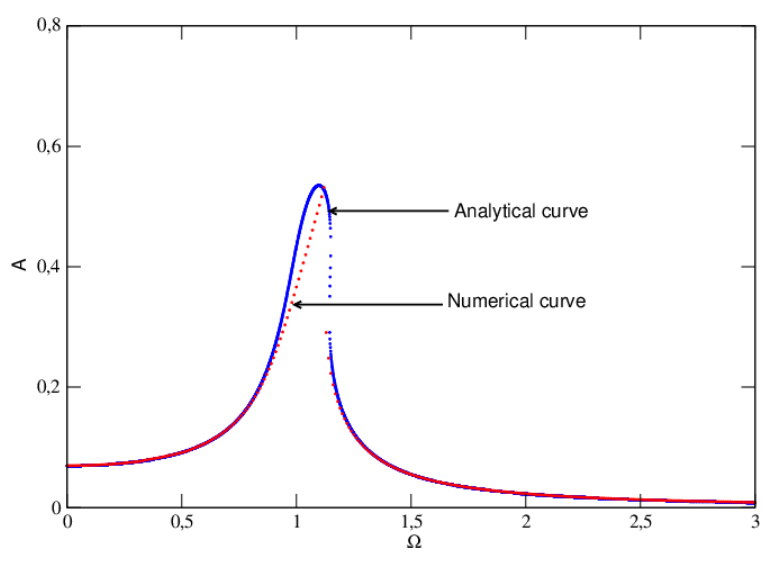

Figure 2: Frequency-response curves with the parameters $\alpha=0, \beta=$ $0.05, k_{1}=0.05, k_{2}=0.05, \mu=0.145, \lambda=1, \gamma=1$ and $F=0.07$.

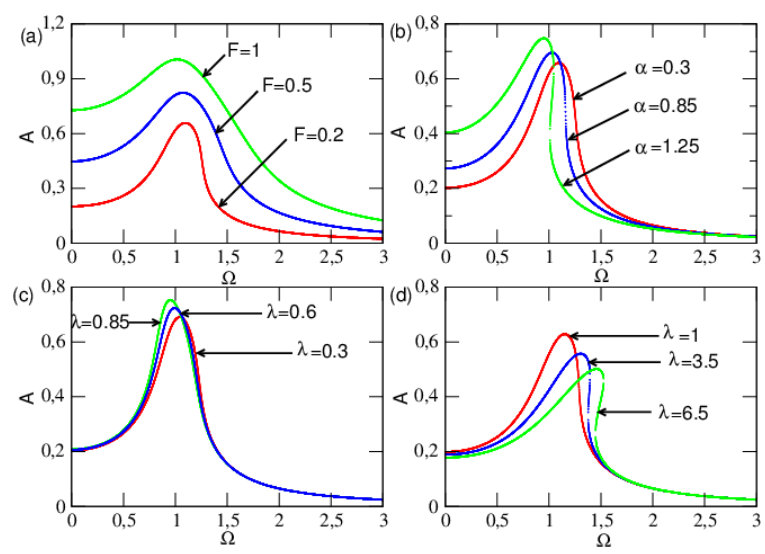

Figure 3: Effects of parameters frequency-response curves with the parameters of Fig.2: $(a)$ effect of $F,(b)$ effect of $\alpha,(c)$ effect of $\lambda$ for $\lambda<1$ and (d): effect of $\lambda$ for $\lambda>1$.

$$
\begin{gathered}
\Gamma_{3}=-\frac{24 \mu A^{2}}{\Omega^{2}}, \quad \Gamma_{4}=\frac{-4 k_{2} A^{2}}{\Omega}, \\
\Lambda_{0}=\gamma+\varepsilon^{2}-\varepsilon \mu+2 k_{2} A^{2}+\frac{3}{2} \lambda A^{2}+\frac{24 \varepsilon \mu A^{2}}{\Omega^{2}}, \\
\Lambda_{1}=\alpha-\varepsilon k_{1}, \quad \Lambda_{2}=\frac{8 \varepsilon \beta A-4 k_{1} A}{\Omega}, \\
\Lambda_{3}=\left(\frac{3 \lambda}{2}-\frac{8 k_{2}}{\Omega^{2}}-\frac{24 \varepsilon \mu}{\Omega^{2}}\right) A^{2}, \quad \Lambda_{4}=\frac{4 \varepsilon k_{2} A^{2}}{\Omega},
\end{gathered}
$$

and

$$
\varepsilon=\frac{\mu}{\Omega}
$$

To further discuss the stability boundaries, we use the following tranformation

$$
\xi=\chi \exp (-\Upsilon \tau) \exp \left[-\frac{1}{2} \int_{0}^{\tau} \Phi\left(\tau^{\prime}\right) d \tau^{\prime}\right]
$$

Thus, we obtain the following standard Hill equation

$$
\begin{aligned}
& \ddot{\xi}+\left(\sigma_{0}+2 \sigma_{1 s} \sin 2 \tau+2 \sigma_{1 c} \cos 2 \tau+2 \sigma_{2 s} \sin 4 \tau+\right. \\
& 2 \sigma_{2 c} \cos 4 \tau+2 \sigma_{3 s} \sin 6 \tau+2 \sigma_{3 c} \cos 6 \tau+2 \sigma_{4 s} \sin 8 \tau+ \\
& \left.2 \sigma_{4 c} \cos 8 \tau\right) \xi=0
\end{aligned}
$$

where

$$
\begin{gathered}
\sigma_{0}=\Lambda_{0}-\Upsilon^{2}-\frac{1}{8}\left(\Gamma_{1}^{2}+\Gamma_{2}^{2}+\Gamma_{3}^{2}+\Gamma_{4}^{2}\right), \\
\sigma_{1 s}=\frac{1}{2}\left(\Lambda_{2}+\Gamma_{1}-\Gamma_{2} \Upsilon-\frac{\Gamma_{1} \Gamma_{4}-\Gamma_{2} \Gamma_{3}}{4}\right),
\end{gathered}
$$



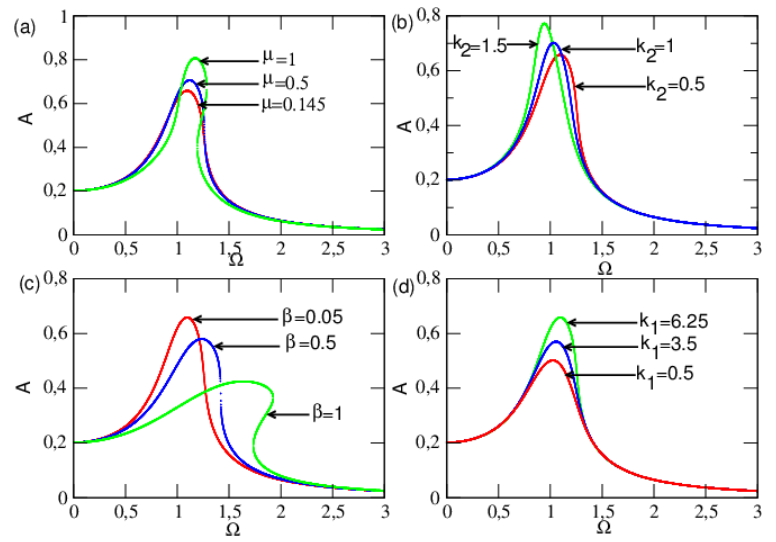

Figure 4: Effects of damping on frequency-response curves with the parameters of Fig.2: $(a)$ effect of $\mu,(b)$ effect of $k_{2},(c)$ effect of $\beta$ and $(d)$ effect of $k_{1}$.

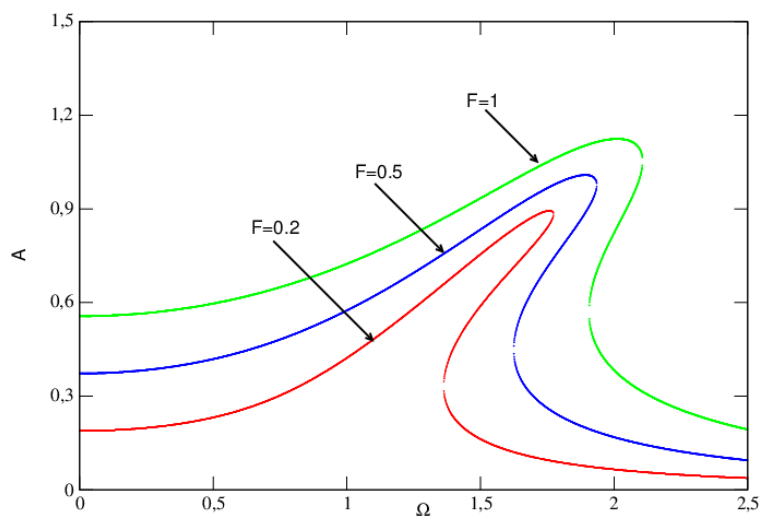

Figure 5: Effects of external forced amplitude on frequency-response curves with $\lambda=3.5$ and other parameters of Fig. 3 .

$$
\begin{gathered}
\sigma_{1 c}=\frac{1}{2}\left(\Lambda_{1}-\Gamma_{2}-\Gamma_{1} \Upsilon-\frac{\Gamma_{1} \Gamma_{3}+\Gamma_{2} \Gamma_{4}}{4}\right), \\
\sigma_{2 s}=\frac{1}{2}\left(\Lambda_{4}+2 \Gamma_{3}-\Gamma_{4} \gamma-\frac{\Gamma_{1} \Gamma_{2}}{4}\right), \\
\sigma_{2 c}=\frac{1}{2}\left(\Lambda_{3}-2 \Gamma_{4}-\Gamma_{1} \Gamma_{3}-\frac{\Gamma_{1}^{2}-\Gamma_{2}^{2}}{8}\right), \\
\sigma_{3 s}=-\frac{\Gamma_{1} \Gamma_{4}+\Gamma_{2} \Gamma_{3}}{8}, \\
\sigma_{3 c}=-\frac{\Gamma_{1} \Gamma_{3}-\Gamma_{2} \Gamma_{4}}{8} \\
\sigma_{4 s}=-\frac{\Gamma_{3} \Gamma_{4}}{8}, \\
\sigma_{4 c}=-\frac{\Gamma_{3}^{2}-\Gamma_{4}^{2}}{16} .
\end{gathered}
$$

From Eq. (11), the stability boundaries are to be investigated around four main parametric resonances defined at $\sigma_{0}=n^{2}(n=1,2,3,4)$. The Floquet theory states $\chi$ may decrease to zero or grow to infinity. Here, the Whittaker method is used to discuss unstable solutions. We assume that the solution of Eq. (11) at the nth unstable region is defined as follows:

$$
\chi=\exp (\kappa \tau) \sin (n \tau-\theta)
$$

with $\kappa$ being the characteristic exponent and $\theta$ a constant. Substituting Eq. (12) into Eq. (11), the characteristic exponent can be written as the following expression:

$$
\kappa^{2}=-\left(\sigma_{0}+n^{2}\right)+\sqrt{4 n^{2} \sigma_{0}+\sigma_{n c}^{2}+\sigma_{n s}^{2}} .
$$

The stability of the process is realized when $\chi(\tau)$ goes to zero as time increases, so that the real part of the quantity $-\Upsilon \pm \kappa$ should be negative since $\chi$ must be real and always positive. Remember that according to Floquet theory, $\Upsilon^{2}>\kappa^{2}$. Thus, the occurence of stable oscillatory states is given as follows :

$$
\begin{aligned}
& v_{n}=\left(\sigma_{0}-n^{2}\right)^{2}+2\left(\sigma_{0}+n^{2}\right) \Upsilon^{2}+ \\
& +\Upsilon^{4}-\left(\sigma_{n c}^{2}+\sigma_{n s}^{2}\right)>0, \quad n=1,2,3,4
\end{aligned}
$$

where $n=1,2,3,4$ represent the first, second, third and fourth parametric resonant state respectively. The fulfillment of the criterion (14) is essential to ensure the stability of a modified RayleighDuffing oscillations. The stability criterion is plotted in Figs.6, 7, 8, 9. The stability criterion is always guaranteed for $n=2,3,4$ as shown in Fig.6. For $n=1$, the system is unstable when $\alpha>0.707$. The stability domain is strongly influenced by the damping parameters (see Figs. 7, 8, 9).
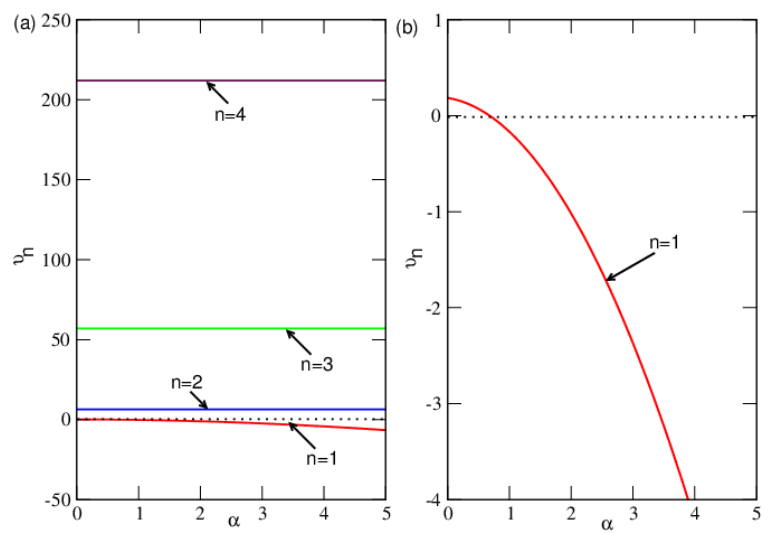

Figure 6: $(a)$ State of the second stability criterion for all the four parametric resonances in the $\left(\alpha, v_{n}\right)$ plane with $\gamma=1, \beta=0.05, k_{1}=0.5, k_{2}=0.5, \mu=$ $0.0005, \lambda=1, A=0.53$ and $\Omega=1.105 ;(b)$ zoom of $(a)$.

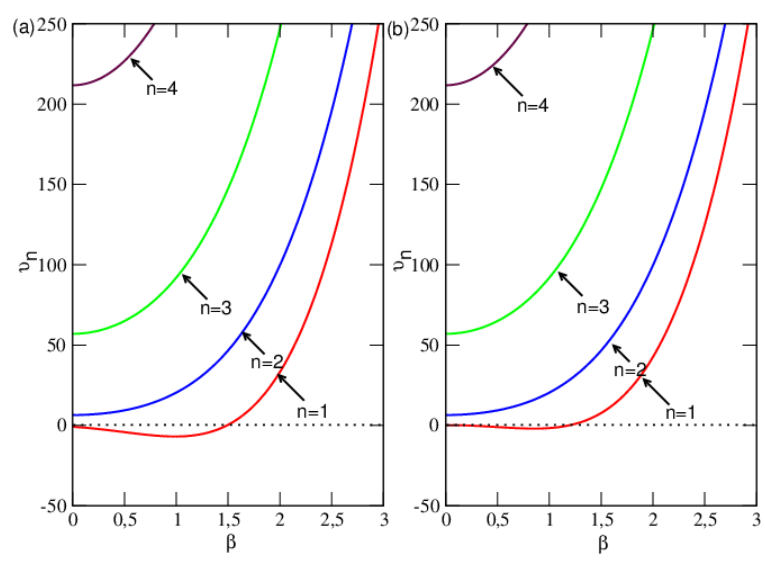

Figure 7: State of the second stability criterion for all the four parametric resonances $\left(\beta, v_{n}\right)$ with the parameters of Fig.6: (a) $\alpha=2.05 ;(b) \alpha=0$.

\section{Bifurcation and transition to chaos}

Our aim in this section is to investigate the way under which chaotic motions arise in the model described by Eq. (1) for resonant states since they are of interest in many phyics phenomena. For this purpose, we numerically solve this equation using the fourth-order 

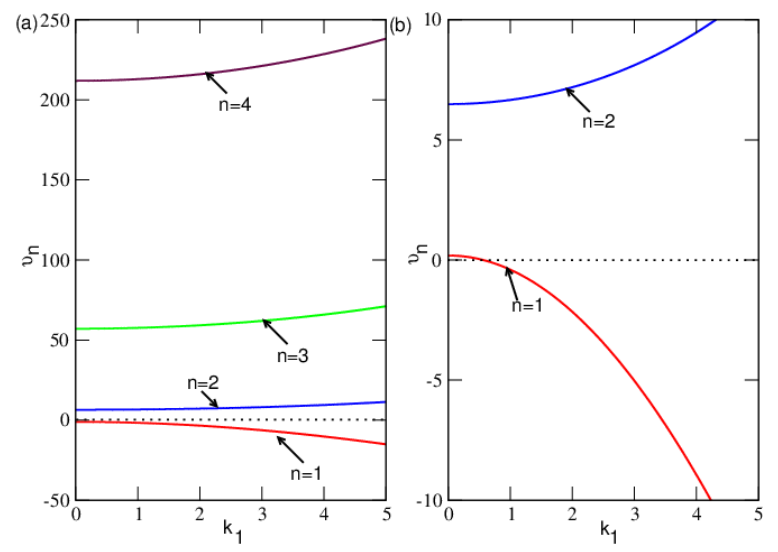

Figure 8: State of the second stability criterion for all the four parametric resonances $\left(k 1, v_{n}\right)$ with the parameters of Fig.6: $(a) \alpha=2.05 ;(b) \alpha=0$.
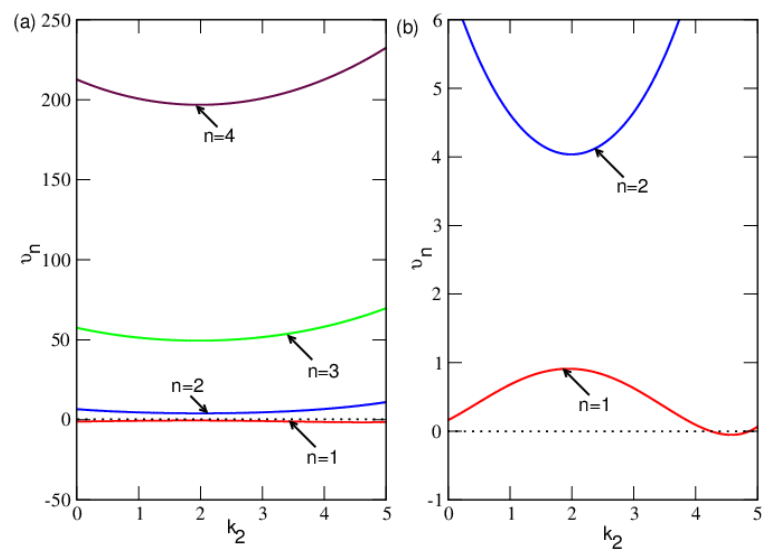

Figure 9: State of the second stability criterion for all the four parametric resonances $\left(k 2, v_{n}\right)$ with the parameters of Fig.6: $(a) \alpha=2.05 ;(b) \alpha=0$.

Runge Kutta algorithm. We plot the resulting bifurcation diagrams and the variation of the corresponding largest Lyapunov exponent as the amplitude $F$ and the nonlinearity parameters varied. The stroboscopic time period used to map various transitions which appear in the model is $T=\frac{2 \pi}{\Omega}$. The largest Lyapunov exponent which is used here as the instrument to measure the rate of chaos in the system is defined as

$L y a=\lim _{t \rightarrow \infty} \frac{\ln \sqrt{d x^{2}+d \dot{x}^{2}}}{t}$

where $d x$ and $d \dot{x}$ are respectively the variations of $x$ and $\dot{x}$. The initial condition which is used here is $\left(x_{0}, \dot{x}_{0}\right)=(0,0)$. From the bifurcation diagram and its corresponding Lyapunov exponents, quasiperiodic and chaotic behaviours are displayed (see Fig. 10). In order to have an idea about the system behaviour as predicted by the bifurcation diagram, various phase portraits for several different values of $F$ chosen in the above mentioned regions are plotted in Fig. 11 using the parameters of Fig. 10. Figs $11(a)$ and $(b)$ represent the phase portrait and its corresponding times histories respectively for $F=0.3$. The portrait phase in this case seems to be like a torus. One might expect to see a quasiperiodic oscillation since the overall torus resembles to the one observed in case of solenoidal attractor (the so-called quasiperiodic attractor). Indeed, by looking at its corresponding time series, one can observe a quasiperiodic state as shown in Fig. $11(b)$. The chaotic oscillation is shown in Figs $11(c)$ and $(d)$ which presented the phase portrait and its corresponding time series respectively for $F=1.15$. For this value of the external force and the parameters of Fig. 11, in order to situate some regions of the initial conditions for which chaotic oscillations are observed, the basin of attraction have been plotted in Fig. 12. In this figure, the blue zone stands for the area where the choice of the initial conditions lead to a chaotic motion while the white area is the domain of periodic or quasiperiodic oscillations. The influences of both the nonlinear unpure quadratic and unpure cubic dissipative parameters and the parametric excitation on the bifurcation sequences and basin of attraction are also investigated, and the results are reported in Figs. 13, 14, 15. Through Figs. 13, 14, we noticed that the quasiperiodic and chaotic oscillations always existed and the chaotic oscillations domain is abundant for high value of the unpure cubic damping coefficient $k_{2}$. The quasiperiodic and chaotic motions domains are highly modified by the amplitude of parametric excitation and unstable oscillations domain become more abundant. Fig. 16 shows the basin of attraction for $k_{1}=0.82, k_{2}=4.73, \alpha=0$ and $\alpha=0.15$. It is observed that the basin of attraction becomes more erode when the unpure quadratic damping $k_{1}$ increases and the unstable oscillations domains increase with $\alpha$.
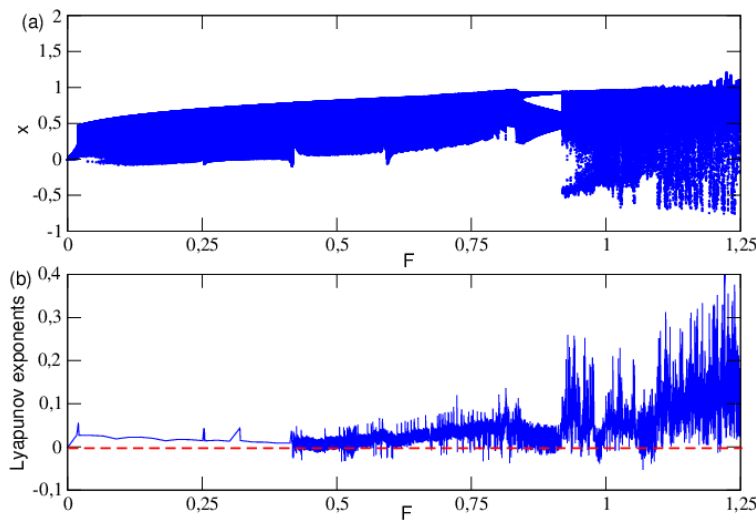

Figure 10: $(a)$ Bifurcation diagram and $(b)$ Lyapunov exponent versus the amplitude $F$ with parameters $\alpha=0.0, \beta=0.05, k_{1}=0.05, k_{2}=4.73, \mu=$ $0.0005, \lambda=1, \gamma=1$ and $\Omega=1.105$.
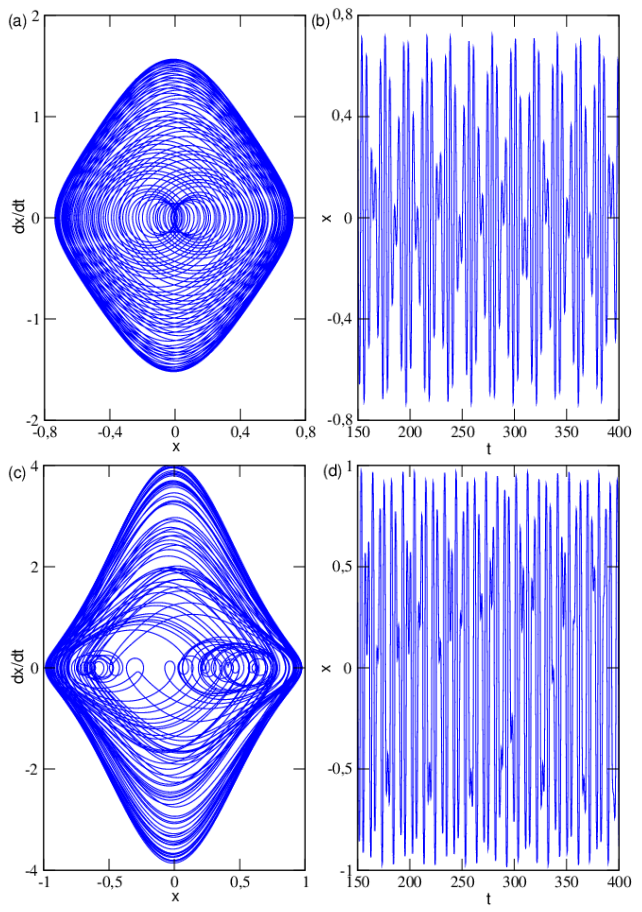

Figure 11: Various phase portraits and corresponding time histories: $(a),(b)$ $F=0.3 ;(c),(d) F=1.15$ and the parameters of Fig.10. 


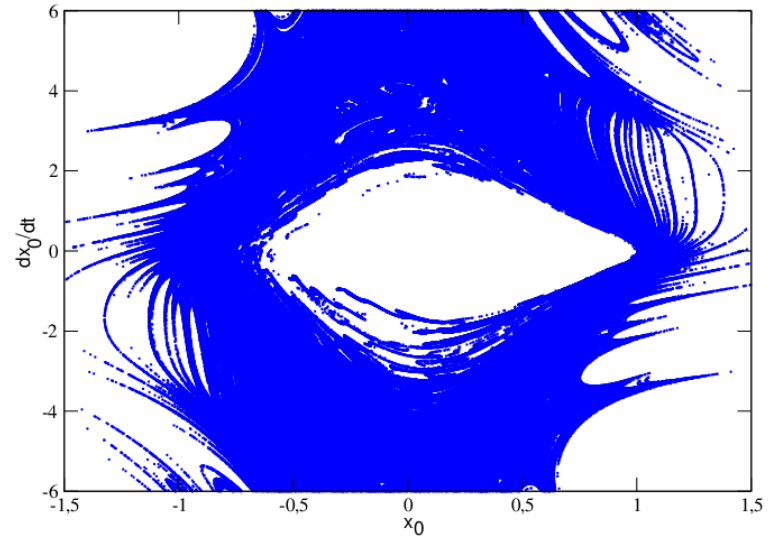

Figure 12: Basin of attraction of the model showing regions of chaotic and non chaotic motions with the parameters of Fig.11 $(c)$.
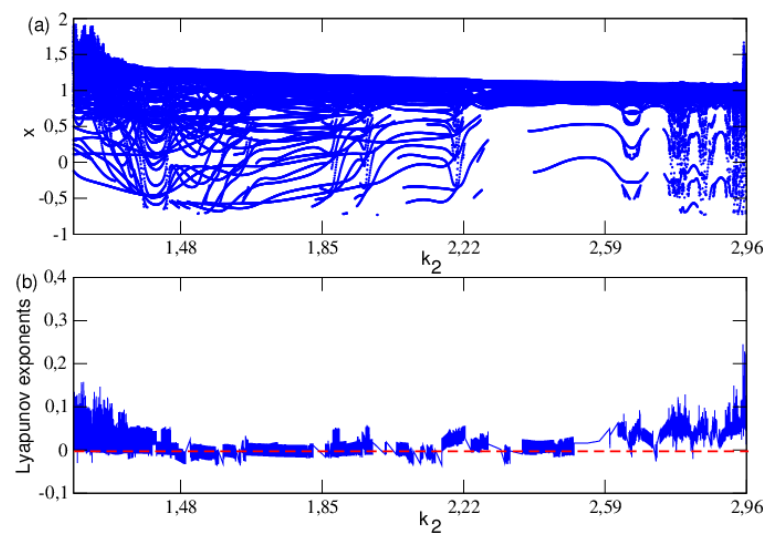

Figure 13: $(a)$ Bifurcation diagram and $(b)$ Lyapunov exponent versus the damping coffecient $k_{2}$ with parameters $\alpha=0.0, \beta=0.05, k_{1}=0.82, \mu=$ $0.0005, \lambda=1, \gamma=1, F=1.15$ and $\Omega=1.105$.

\section{Conclusion}

In this work, regular and chaotic behaviours of a physical system modelled by a modified Rayleigh-Duffing oscillator are investigated. The originality of the work is related to the potential used, the analytical methods employed and then to the simultaneous consideration into account of new nonlinear cubic, pure quadratic and hybrid dissipative terms which modify the classical Rayleigh-Duffing oscillator. It is obtained the hysteresis and jump phenomena and resonance phenomenon appeared in $(\Omega, A)$ space. It is found that the nonlinear damping and parametric excitation amplitude affected the amplitude and frequency of resonance. The two loci of jump points equation has been obtained indicating the jump phenomenon domain evolution. It is also found the second stability criterion of harmonic oscillations of the modified Rayleigh-Duffing oscillator by using the Floquet theory and Whittaker method and the effects of the damping parameters on the unstable domains have been strongly studied. Various bifurcation structures showing different types of transitions from quasiperiodic motions to chaotic motions have been drawn and the influences of different parameters on these motions have been pointed out. It is noticed that chaotic motion is controlled by the parameters $k_{1}, k_{2}, \alpha$. The results of basin of attraction show a way to predict initial conditions in which regular and chaotic behaviours are obtained. This could be helpful for experimentalists whose interest is to try to stabilize such a system with differents parameters or initial conditions. For practical interests, it is useful to develop tools and to find the ways to control or suppress such undesirable regions. This will be also useful to control high amplitude of oscillations obtained
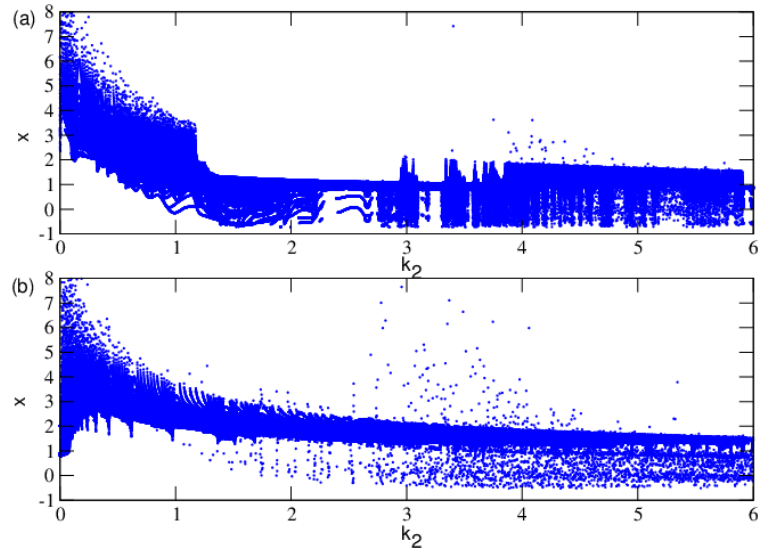

Figure 14: Bifurcation diagram versus the damping coffecient $k_{2}$; (a) $\alpha=0.0,(b) \alpha=0.15$ with others parameters $\beta=0.05, k_{1}=0.82, \mu=$ $0.0005, \lambda=1, \gamma=1, F=1.15$ and $\Omega=1.105$.
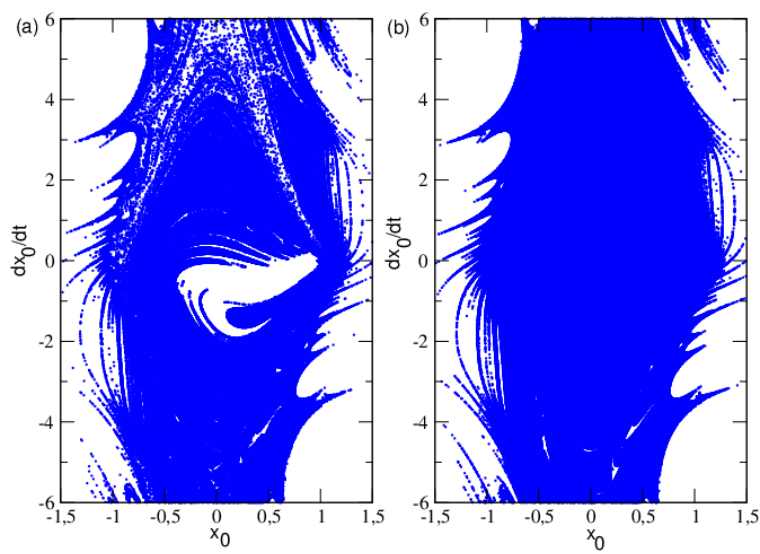

Figure 15: Basin of attraction of the model showing regions of chaotic and non chaotic motions with the parameters of Fig. 14.

and which are generally source of instability in systems modeled by the modified Rayleigh-Duffing oscillator equation.

\section{Acknowlegments}

The authors thank Drs Hervé Enjieu Kadji, Laurent Hinvi and Professor Paul Woafo for their fruitfull suggestions and their collaboration. We would also like to thank very much the anonymous referees whose useful criticisms, comments and suggestions would help strengthen the content and the quality of the paper.

\section{References}

[1] Carrol, T. L., Communicating With Use of filtered, Synchronized, Chaotic Signals. Fundam. Theory Appl. (1995);42:105.

[2] Nayfeh, A. H. and Mook, D. T., Nonlinear Oscillations. Wiley, New York (1979).

[3] Wang, B., Barcilon, A. and Fang, Z., Stochastic Dynamics of El NinoSouthern Oscillation, Journal of Atmospheric Sciences (1996) 56:5-23.

[4] Francescutto, A. and Contento, G., Bifurcations in ship rolling: experimental results and parameter identification technique. Ocean Engineering, (1999); 26: 1095-1123.

[5] Hayashi, C., Nonlinear Oscillations in Physical Systems. McGrawHill, New York (1964).

[6] Rand, R. H., Ramani, D. V, Keith, W. L. and Cipolla, K. M., The quadratically damped Mathieu equation and its application to sub marine dynamics. Control of Vibration and Noise: New Millennium (2000); 61: 39-50.

[7] Zalalutdinov, M., Parpia, J.M., Aubin, K.L., Craighead, H.G., T.Alan, Zehnder, A.T. and Rand, R.H., Hopf Bifurcation in a Disk-Shaped NEMS, Pro-ceedings of the 2003 ASME Design Engineering Technical 

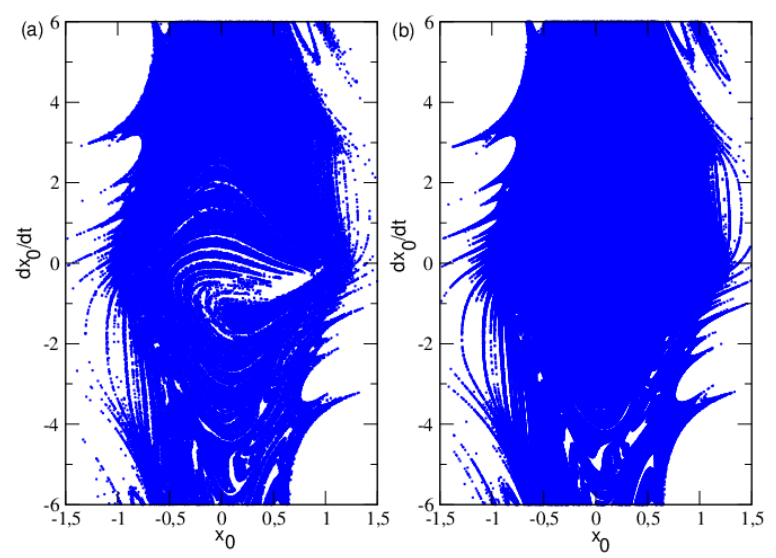

Figure 16: Effect of unpure quadratic damping on basin of attraction of the model showing regions of chaotic and non chaotic motions with the parameters of Fig.14.

Conferences, Biennial Conference on Mechanical Vibrations and Noise, Chicago, IL, Sept. 2-6, (2003); 56: 5-23.

[8] Zalalutdinov, M., Olkhovets, A., Zehnder, A., Ilic, B., Czaplewski, D. and Craighead, H. G. Optically pumped parametric amplification for micro-mechanical systems, Applied Physics Letters (2008); 78: 3142-3144

[9] Soliman, M. S. and Thompson, J. M. T., The effect of damping on the steady state and basin bifurcation patterns of a nonlinear mechanical oscillator. Int. J. Bifurcation and Chaos 1992); 2: 81-92.

[10] Wirkus, S., Rand, R. H. and Ruina, A., How to pump a swing, The College Mathematics Journal (1998) 29:266-275.

[11] Ostrikov, K. and Xu, S., Plasma-aided Nanofabrication: from Plasma Sources to Nanoassem-bly, John Wiley Sons, Weinheim (2007).

[12] Yamapi, R., Aziz-Alaoui, M. A., Vibration analysis and bifurcations in the self-sustained electromechanical system with multiple functions, Communications in Nonlinear Science and Numerical Simulation (2007) 12:1534-1549.

[13] Miwadinou, C.H., Monwanou, A. V. and Chabi Orou, J. B., Active Control of the Parametric Resonance in the Modified Rayleigh-Duffing Oscillator, African Review of Physics (2O14); 9: 227-235.

[14] Miwadinou, C. H., Monwanou, A. V. and Chabi Orou, J. B., Effect of Nonlinear Dissipation on the Basin Boundaries of a Driven Two-Well Modified Rayleigh-Duffing Oscillator. Int. J. Bifurcation and Chaos (2015); 2: 1550024 .

[15] Miwadinou, C. H., Hinvi, A. L, Monwanou, A. V. and Chabi Orou, J. B., Nonlinear dynamics of a $\phi^{6}-$ modified Duffing oscillator: resonant oscillations and transition to chaos. Nonlinear Dyn. (2017); 88:97-113.

[16] Miwadinou, C. H., Hinvi, A. L, Monwanou, A. V., Koukpemedji, A. A., Ainamon, C. and Chabi Orou, J. B., Melnikov Chaos in a Modified Rayleigh-Duffing Oscillator with $\phi^{6}-$ Potential. Int. J. Bifurcation and Chaos (2016); 5: 1650085.

[17] Pandey, M., Rand, R. and Zehnder, A., Perturbation Analysis of Entrainment in a Micromechanical Limit Cycle Oscillator, Communications in Nonlinear Science and Numerical Simulation (2006).

[18] Morrison, T. M., Three Problems in Nonlinear Dynamics With 2:1 Parametric Exitation. Ph.D. Cornell University (2006).

[19] Wang, B. and Fang, Z., Chaotic Oscillations of Tropical Climate: A Dynamic System Theory for ENSO. Journal of Atmospheric Sciences (1996); 53:2786-2802.

[20] K.W. Holappa, J.M. Falzarano Application of extended state space to nonlinear ship rolling, Ocean Engineering (1999); 26: 227-240.

[21] Enjieu, K. H. G., Nana, N. B. R. , Orou, C. J. B. and Talla, P. K. Nonlinear dynamics of plasma oscillations modeled by an anharmonic oscillator. Phys. Plasmas, (2008);15:1-13.

[22] Enjieu, K. H. G. and Nana, N. B. R., Nonlinear dynamics of plasma oscillations modeled by an anharmonic oscillator. Commun. Nonlinear Sci. Numer. Simulat., (2012);17:1779-1794.

[23] Nayfeh, A. H., Introduction to perturbation techniques, John Wiley and Sons, New York (1981). 